\title{
Neonatal sepsis in a tertiary unit in South Africa
}

Dharshni Pillay ${ }^{1,2^{*}}$, Lerusha Naidoo ${ }^{3,4}$, Khine Swe Swe-Han ${ }^{1,2}$ and Yesholata Mahabeer ${ }^{1,2}$

\begin{abstract}
Background: Antimicrobial resistance (AMR) has emerged as a global threat to healthcare resulting in an increase in morbidity and mortality. Neonatal sepsis is ranked as the third highest cause of neonatal demise globally, in which AMR accounted for 31.0\% of deaths. AMR in neonates has been poorly characterised in Durban, South Africa. Thus, the resultant effect of AMR on empiric regimens for neonatal sepsis is uncertain in this setting. Therefore, this study analysed the aetiology and antimicrobial susceptibility patterns of bloodstream infections within the neonatal intensive care unit at a tertiary hospital in Durban, with the aim of establishing an effective empiric regimen for the unit.

Methods: A retrospective data review on positive blood cultures from the neonatal intensive care unit at Inkosi Albert Luthuli Central Hospital was conducted. Three time periods were analysed: 2014, 2016 and 2018. Culture data from neonates aged 0-30 days were included and repeat cultures were de-duplicated. The frequency of common organisms and their antimicrobial susceptibilities were analysed. Fischer's exact test was used for subgroup analysis. Poisson and logistic regressions were used to assess significant trends in organisms and antimicrobial susceptibilities over time.

Results: Late-onset sepsis (86.8\%) predominated over early-onset sepsis (13.2\%). A preponderance of gram-positive organisms (68.7\%) over gram-negatives (26.8\%) and fungi (4.5\%) was detected. Common pathogens included coagulase-negative staphylococci (53.5\%), Klebsiella pneumoniae (11.6\%), enterococci (9.3\%), and Acinetobacter baumannii (7.7\%). Despite the small contribution of fungi to the microbial profile, fluconazole-resistant Candida parapsilosis predominated within that group. High rates of resistance to first- and second-line antibiotics were also noted among gram-positive and gram-negative organisms. Multidrug resistant organisms included extendedspectrum beta-lactamase (ESBL) K. pneumoniae (7.6\%) and extensively-drug resistant A. baumannii (7.0\%). However, a statistically significant decrease in ESBL-producing organisms was documented during the entire study period $(p=0.005)$.

(Continued on next page)
\end{abstract}

\footnotetext{
* Correspondence: dharshnipillay@gmail.com

${ }^{1}$ Department of Medical Microbiology, National Health Laboratory Service, Inkosi Albert Luthuli Central Hospital, 800 Vusi Mzimela Road, Durban, KwaZulu-Natal 4091, South Africa

${ }^{2}$ School of Laboratory Medicine and Medical Sciences, Nelson R. Mandela School of Medicine, University of KwaZulu Natal, 716 Umbilo Road, Berea, KwaZulu-Natal 4001, South Africa

Full list of author information is available at the end of the article
}

(c) The Author(s). 2021 Open Access This article is licensed under a Creative Commons Attribution 4.0 International License, which permits use, sharing, adaptation, distribution and reproduction in any medium or format, as long as you give appropriate credit to the original author(s) and the source, provide a link to the Creative Commons licence, and indicate if changes were made. The images or other third party material in this article are included in the article's Creative Commons licence, unless indicated otherwise in a credit line to the material. If material is not included in the article's Creative Commons licence and your intended use is not permitted by statutory regulation or exceeds the permitted use, you will need to obtain permission directly from the copyright holder. To view a copy of this licence, visit http://creativecommons.org/licenses/by/4.0/. The Creative Commons Public Domain Dedication waiver (http://creativecommons.org/publicdomain/zero/1.0/) applies to the data made available in this article, unless otherwise stated in a credit line to the data. 
(Continued from previous page)

Conclusions: It was determined that first-line antimicrobials, advocated by the World Health Organization for treatment of neonatal sepsis, proved ineffective in this unit due to high levels of AMR. Therefore, this study advises that meropenem with or without vancomycin provides optimal empiric cover. Amphotericin B is advocated for empiric antifungal therapy. Ongoing surveillance is necessary.

Keywords: Neonatal sepsis, Microbial profiles, Antimicrobial resistance, Empiric regimens

\section{Introduction}

Antimicrobial resistance (AMR) has emerged as a global threat to healthcare resulting in an increase in morbidity and mortality [1]. An estimated $31.0 \%$ of deaths from neonatal sepsis are attributed to AMR [2]. Sepsis accounts for $6.8 \%$ of neonatal deaths, ranking it as the third highest cause of neonatal demise following preterm births and intrapartum-related events [3]. In SubSaharan Africa, sepsis-related neonatal mortality rates are high and range between 17.0 to $29.0 \%$ [4].

Neonatal sepsis presents unique diagnostic challenges largely due to the absence of a universal definition [5]. Traditionally defined as sepsis within the first 28 days of life, neonatal sepsis can be further stratified into early-onset $(<3$ days) and late-onset $(\geq$ 3 days) [6]. It entails a collection of non-specific clinical features or laboratory signs of sepsis with positive microbiological cultures from a sterile sample (although cultures may sometimes be negative). A significant isolate from a blood culture is the gold standard for diagnosis [6].

Based on blood culture data, early-onset sepsis (EOS) and late-onset sepsis (LOS) differ in microbial profiles. Group B streptococcus (GBS) is a major cause of early onset sepsis in high-income countries (HIC) [7]. This contrasts with the bacteriological profile of resourcelimited settings where GBS rates are lower and Klebsiella pneumoniae, Staphylococcus aureus and coagulasenegative staphylococci (CoNS) are the predominant pathogens of EOS $[8,9]$. In South Africa, EOS is mainly caused by GBS, K. pneumoniae and Escherichia coli [1012].

A wider spectrum of gram-negatives, including $K$. pneumoniae, Citrobacter species, E. coli, Enterobacter species, Acinetobacter baumannii and Pseudomonas aeruginosa are observed in LOS [13, 14]. Furthermore, CoNS cause significant cases of gram-positive LOS in many countries $[11,15]$. Other important bacterial causes of LOS in South Africa are enterococci, K. pneumoniae and Acinetobacter species [12, 16].

A strong association between fungal infections and LOS suggests a causal relationship with hospital intervention [17]. Globally, Candida species have been reported as the third leading cause of blood-stream infection (BSI) amongst extremely-low-birthweight neonates and in South Africa, C. parapsilosis is a leading cause of candidaemia in neonates [18].

Global resistance to first-line empiric treatment regimens is on the increase $[8,13,19]$. This NICU recommends empiric antibiotic regimens according to timing of sepsis. Amoxicillin-clavulanate is used for EOS and piperacillin-tazobactam plus amikacin for LOS. Escalation to meropenem depends on the therapeutic response with the addition of vancomycin and fluconazole in the presence of risk factors.

Multi-drug resistance adds a further complication to antimicrobial choices. South African studies have documented the emergence of drug resistance to multiple antibiotics, including extended-spectrum $\beta$-lactamase production (ESBL) and methicillin-resistant S. aureus (MRSA) in neonates [10, 12, 16, 20]. These changing patterns of resistance require regular microbial surveys be conducted. Consequently, understanding the microbial profile of a neonatal unit contributes significantly to early appropriate empiric antimicrobial choices in the management of sepsis. This improves therapeutic outcomes and reduces mortality. Notably in KwaZulu-Natal (which encompasses Durban), a limited scientific data base surrounding local microbial profiles and AMR in neonatal sepsis exists. Therefore, this study aims to establish the microbiological and antimicrobial susceptibility profiles and trends of neonatal bloodstream infections in the NICU of Inkosi Albert Luthuli Central Hospital to guide empiric antimicrobial management of EOS and LOS.

\section{Methods}

\section{Study design, location, and period}

This study is a retrospective review of positive blood cultures from the NICU at IALCH, which is a tertiary and the only quaternary referral unit for the province of KwaZulu-Natal, South Africa. The unit consists of 12 intensive care and 8 high-care beds, with approximately 700 to 800 admissions per year and a bed occupancy rate of $100 \%$. The patients are usually from surgical, neurosurgical and cardiology disciplines.

Data was collected from 2014 to 2018 at three biennial periods: 2014, 2016 and 2018. The data was accessed from the National Health Laboratory Service (NHLS) Central Data Warehouse. 


\section{Study population}

The study samples consisted of all positive blood cultures from the NICU for the period January to December in years 2014, 2016 and 2018. Individual episodes of sepsis were sought for the study population. Samples were included from patients aged 0 to 30 days of life. Repeat blood cultures taken within 14 days of the index culture, where the same organism was isolated again, were excluded from the study.

\section{Laboratory methods and information handling}

The general techniques utilised by the laboratory to generate the microbial identifications and antimicrobial susceptibility testing include manual methods such as Kirby-Bauer disk diffusion. Automated identifications and susceptibility testing were performed using Vitek 2 Advanced Expert System $^{\mathrm{Tx}}$ (bioMerieux). Data was entered onto the laboratory information system which interfaces with the Central Data Warehouse.

\section{Measurements}

The onset of sepsis was classified as early-onset $(<3$ days old) and late-onset sepsis ( $\geq 3$ days old) measured from the date of birth until collection of the index culture.

Primary outcomes:

1. The frequency of common organisms and antimicrobial susceptibility patterns were evaluated as a proportion of the total number of positive cultures. Antimicrobial susceptibility patterns were stratified and analysed according to gram-positive, gram-negative, and fungal antimicrobial panels. These panels consisted of antimicrobials routinely tested within the IALCH microbiology laboratory.

2. Trends in prevalence of organisms and antimicrobial susceptibilities per year were calculated and prevalence of specific multi-drug resistant (MDR) organisms during the study period was determined. These MDR organisms included [21]:

- MDR gram-negatives - resistant to at least one agent from two or more classes of all tested antimicrobial agents.

- XDR - non-susceptibility to one or more agents in two or less categories of antimicrobials.

- Carbapenem-resistant Enterobacterales (CRE) resistant to at least one carbapenem (imipenem, meropenem, ertapenem).

- ESBL - resistant to a third or fourth generation cephalosporins or detected as an ESBL through automated methods (Vitek 2 Advanced Expert System $^{\text {Tw" }}$, bioMerieux)

- MRSA - S. aureus resistant to cloxacillin.

- VRE - enterococci resistant to vancomycin.

\section{Statistical analysis}

Descriptive statistics were used to summarise the data. Categorical data were summarised by frequencies and percentage. The frequency of selected organisms was reported by year. Susceptibility of each drug was reported as the percentage susceptible. Frequency was calculated as a measure of the total number of samples in the data series. The number of each organism seen per year is a count variable. Comparisons of pathogens by subgroup, such as early-onset and late-onset neonatal sepsis, was done using Chi Square or Fisher's exact test. Temporal trends in the number of organisms and antimicrobial susceptibility patterns over time were analysed using Poisson and logistic regression. Stata V13.1 was used for the data analysis and $p$-value of 0.05 was considered statistically significant.

\section{Ethical considerations}

This study has received approval from the University of KwaZulu-Natal Biomedical Research Ethics Committee (BE019/19), IALCH, NHLS and the KwaZulu-Natal Department of Health.

\section{Results}

As demonstrated in Figs. 1, 1607 isolates were initially assessed for eligibility during the three study periods. The final analysis included 681 isolates from 3 years: 2014 (207 isolates), 2016 (222 isolates) and 2018 (252 isolates). These were stratified into 3 groups: grampositives (S. aureus, CoNS, Streptococcus species, Enterococcus species), gram- negatives (Enterobacterales, nonfermenting gram-negative organisms) and fungi.

\section{Overall microbial profile}

Gram-positive organisms were predominantly isolated in this study $(468 / 681 ; 68.7 \%)$. Within the gram-positive category, the main organisms were CoNS (363/468; $77.6 \%)$, Enterococcus species $(64 / 468 ; 13.7 \%)$, S. aureus (24/468; 5.1\%) and Streptococcus species (17/468; 3.6\%). Often CoNS are common blood culture contaminants and in the absence of clinical correlation the significance of these organisms is unclear. If CoNS were excluded from the analysis enterococci emerge as the leading cause of gram-positive sepsis $(64 / 105 ; 61.0 \%)$, comprising Enterococcus faecium (40/64, 62.5\%); Enterococcus faecalis $(22 / 64 ; 34.4 \%)$ and Enterococcus species (2/64; $3.1 \%)$. Only one Streptococcus agalactiae isolate was found during the study period. 
Samples assessed for eligibility $(n=1607)$

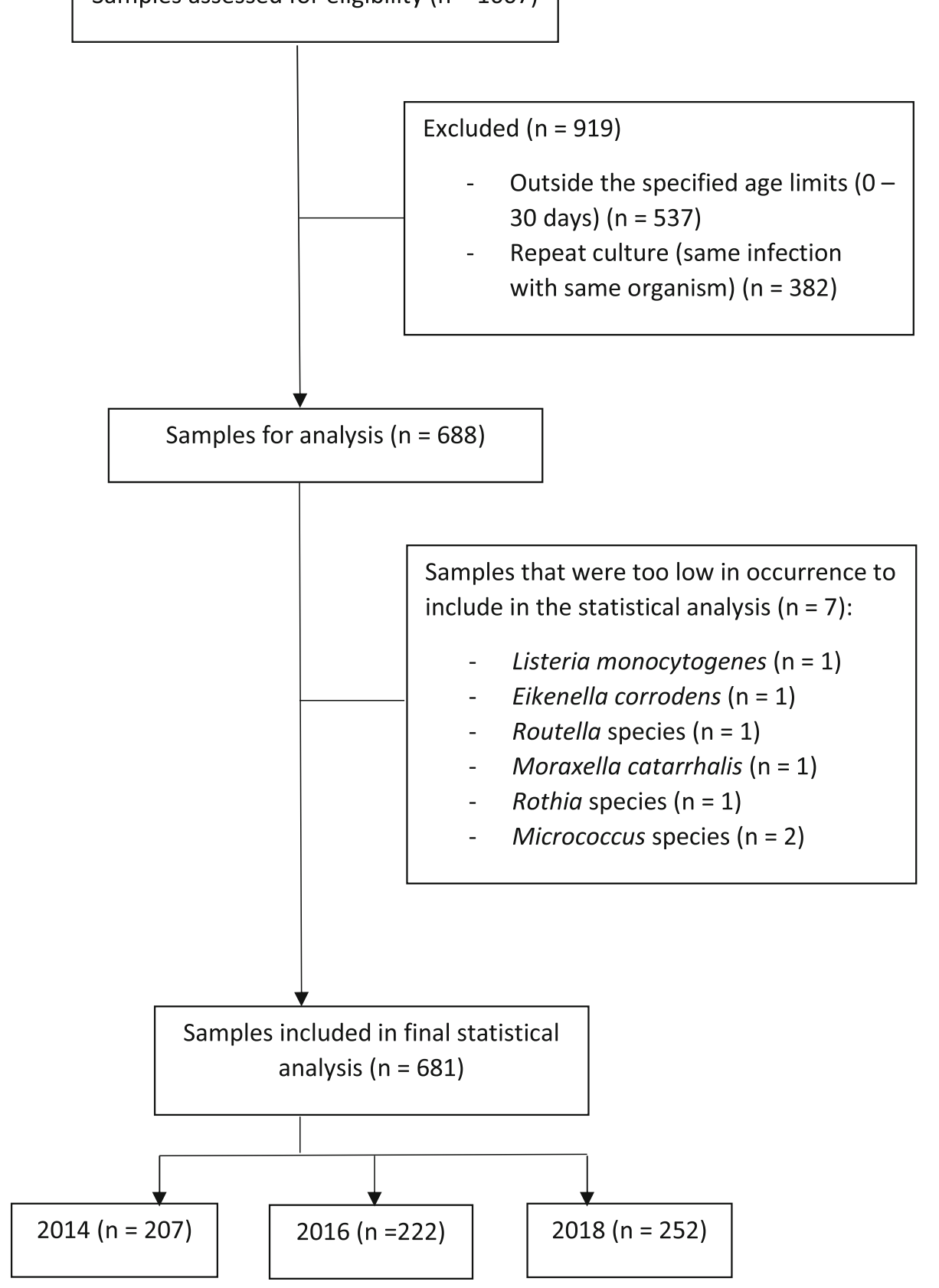

Fig. 1 Derivation of study sample

Gram-negatives accounted for $26.8 \%$ of the total study population (182/681) which were divided into Enterobacterales $(118 / 182 ; 64.8 \%)$ and non-fermenters $(64 / 182$; $35.1 \%)$. Within the Enterobacterales family, the most common organisms were $K$. pneumoniae (79/118; $66.9 \%)$, E. coli $(13 / 118 ; 11.0 \%)$ and Serratia marcescens (10/118; 8.5\%). Acinetobacter baumannii (53/64; 82.8\%) accounted for greater than $80 \%$ of non-fermenters followed by Stenotrophomonas maltophilia (6/64; 9.4\%) and Pseudomonas aeruginosa (5/64; 7.8\%).

Fungal isolates were less commonly isolated than bacterial isolates (4.5\% vs. $95.5 \%)$ and consisted of C. parapsilosis (14/31; 45.2\%), C. albicans $(9 / 31 ; 29.0 \%)$ and other Candida species that were not speciated further (8/31; 25.8\%). Non-albicans Candida species (22/31; $71.0 \%)$ predominated over C. albicans (9/31; 29.0\%). 
Microbial profile trends over 2014, 2016 and 2018

As summarised in Table 1, coagulase-negative staphylococci (53.2\%), K. pneumoniae (11.5\%), A. baumannii (7.8\%) and E. faecium (5.9\%) were the leading organisms across the study period and predominated within each analysed year. An increase in the number of enterococci (E. faecium and E. faecalis) was observed over time, and although not statistically significant, by 2018 , it was the commonest organism in the unit after CoNS. Analysis of the trends of the other organisms revealed no significant patterns, except for Streptococcus species. Streptococcus species, specifically viridans streptococci, demonstrated a significant increase in the number of isolates between 2014 and 2018 (IRR 9. 04; 95\% CI [1.17, 69.99]; $p=$ 0.04). However, their overall contribution to the sample pool was low, $16 / 681$ (1/16 in 2014, 4/16 in 2016 and $11 / 16$ in 2018), therefore, there was no impact on the leading pathogens over the three study years.

\section{Early-onset sepsis versus late-onset sepsis}

In this study, the majority of organisms were isolated during late-onset sepsis (591/681, 86.8\%), with earlyonset sepsis (EOS) accounting for only $13.2 \%(90 / 681)$ of cases $(p=0.02)$. As stipulated in Table 2, S. aureus and $E$. faecalis were more significant in EOS ( $p=0.006$ and $p=0.048$, respectively). E. faecium emerged as an important gram-positive organism of LOS $(p=0.2)$. In addition, although $A$. baumannii is a predominant gram-negative organism in EOS, more $K$. pneumoniae isolates emerged in LOS. However, this difference was not of statistical significance.

\section{Overall antimicrobial susceptibility}

Among the gram-positive organisms, the susceptibilities to first-line therapies (i.e., ampicillin and cloxacillin) were low. However, all gram-positive isolates tested

Table 1 Trend in leading pathogens over the three study periods $(2014,2016,2018)$

\begin{tabular}{llll}
\hline Year & Rank & Organisms & Percentage \\
\hline $\mathbf{2 0 1 4}$ & 1 & CoNS & 53.0 \\
& 2 & K. pneumoniae & 14.4 \\
& 3 & A. baumannii & 9.0 \\
$\mathbf{2 0 1 6}$ & 4 & Enterococcus species & 9.0 \\
& 1 & CoNS & 51.4 \\
& 2 & K. pneumoniae & 16.7 \\
& 3 & Enterococcus species & 7.6 \\
$\mathbf{2 0 1 8}$ & 4 & A. baumannii & 6.7 \\
& 1 & CoNS & 55.2 \\
& 2 & Enterococcus species & 11.5 \\
& 3 & K. pneumoniae & 9.9 \\
& 4 & A. baumannii & 5.1 \\
\hline
\end{tabular}

Table $\mathbf{2}$ Leading pathogens in early-onset sepsis versus lateonset sepsis

\begin{tabular}{llllll}
\hline & \multicolumn{2}{l}{ Early-onset sepsis $(<$} & $\mathbf{3}$ days $)$ & & Late-onset sepsis $(\geq \mathbf{3}$ days $)$ \\
\cline { 2 - 3 } Rank & Organism & Percent & & Organism & Percent \\
\hline $\mathbf{1}$ & CoNS & 56.7 & & CoNS & 52.8 \\
$\mathbf{2}$ & A. baumannii & 7.8 & & K. pneumoniae & 12.4 \\
$\mathbf{3}$ & K. pneumoniae & 6.7 & & A. baumannii & 7.8 \\
$\mathbf{4}$ & E. faecalis & 6.7 & & E. faecium & 6.6 \\
$\mathbf{5}$ & Staphylococcus aureus & 6.7 & & \\
\hline
\end{tabular}

during the study period were susceptible to vancomycin (100.0\%).

Gram-negatives also demonstrated high levels of resistance to commonly used first- and second-line antibiotics. Enterobacterales susceptibility to cefotaxime (34.2\%) and piperacillin-tazobactam (55.5\%) was low. However, susceptibility to carbapenems was maintained at greater than $90.0 \%$. Additionally, this order demonstrated a higher susceptibility to amikacin than gentamicin (70.3\% vs $39.7 \%$, respectively).

Analysis of the non-fermenters, demonstrated low levels of susceptibility towards all tested antibiotics, including third-line agents: meropenem (17.2\%), imipenem (13.5\%) and ciprofloxacin (19.7\%). The highest susceptibility was noted for amikacin (49.1\%).

Approximately half the fungal isolates tested were susceptible to fluconazole, the first-line antifungal agent used in the unit.

Table 3 demonstrates the antimicrobial susceptibilities analysed over the study period.

\section{Antimicrobial susceptibility trends over 2014, 2016 and 2018}

Susceptibility during 2014, 2016 and 2018, for ciprofloxacin, ceftazidime, imipenem, meropenem, penicillin, ampicillin, piperacillin/tazobactam, vancomycin was static. However, a combined analysis of all the gramnegatives demonstrated a significant decrease in susceptibility of amikacin between 2014 (85.8\%), 2016 (65.0\%) and 2018 (53.8\%) (OR 0.24; 95\% CI [0.10, 0.59]; $p=$ 0.002). During the same period, there was an increase in gentamicin susceptibility (19.7 to $43.4 \%$; OR 3.14 ; $95 \%$ CI $[1.33,7.42] ; p=0.01)$. Another notable finding was a statically significant increase in cefotaxime susceptibility for Enterobacterales between 2014 (24.4\%), 2016 (21.7\%) and 2018 (55.1\%) (OR 3.29; 95\% CI [1.15, 7.09]; $p=$ 0.02).

\section{Multidrug resistant organisms}

Figure 2 describes the composition of the MDROs, which constituted $20.0 \%$ of the total sample population $(138 / 681)$. 
Table 3 Overall antimicrobial susceptibility and trends for 2014, 2016 and 2018

\begin{tabular}{|c|c|c|c|c|c|}
\hline Antimicrobials & $\begin{array}{l}2014 \\
\text { n (\%) }\end{array}$ & $\begin{array}{l}2016 \\
\text { n (\%) }\end{array}$ & $\begin{array}{l}2018 \\
\text { n (\%) }\end{array}$ & Total study period - $\mathrm{n}(\%)$ & Episodes omitted $^{a}(n)$ \\
\hline \multicolumn{6}{|l|}{ Gram positive organisms } \\
\hline \multicolumn{6}{|l|}{ Staphylococcus aureus } \\
\hline Cloxacillin & $0 / 4(0.0)$ & $4 / 10(40.0)$ & $1 / 10(10.0)$ & $5 / 24(20.8)$ & 0 \\
\hline Vancomycin & $3 / 3(100.0)$ & $10 / 10(100.0)$ & 9/9 (100.0) & 22/22 (100.0) & 2 \\
\hline \multicolumn{6}{|l|}{ CoNS } \\
\hline Cloxacillin & $5 / 100(4.5)$ & $13 / 114(10.4)$ & 14/139 (11.1) & $32 / 363(8.8)$ & 0 \\
\hline Vancomycin & $11 / 11(100.0)$ & 14/14 (100.0) & 21/21 (100.0) & $46 / 46(100.0)$ & 317 \\
\hline \multicolumn{6}{|l|}{ Enterococcus species } \\
\hline Ampicillin & 6/18 (33.3) & $7 / 17(41.2)$ & $11 / 29(37.9)$ & 24/64 (37.5) & 0 \\
\hline Vancomycin & 18/18 (100.0) & 17/17 (100.0) & 29/29 (100.0) & 64/64 (100) & 0 \\
\hline \multicolumn{6}{|l|}{ Gram negatives organisms } \\
\hline \multicolumn{6}{|l|}{ Enterobacterales } \\
\hline Amikacin & $37 / 41(90.2)$ & 30/43 (38.6) & 27/33 (57.6) & $94 / 117(80.3)$ & 1 \\
\hline Cefotaxime & 10/41 (24.4) & $13 / 43(30.2)$ & $17 / 33(51.5)$ & 40/117 (34.2) & 1 \\
\hline Ceftazidime & $10 / 40(25.0)$ & 13/43 (30.2) & 18/33 (54.5) & $41 / 116(35.3)$ & 2 \\
\hline Ciprofloxacin & 22/41 (53.7) & $31 / 44(70.5)$ & 20/32 (62.5) & 73/117 (62.4) & 1 \\
\hline Ertapenem & 33/34 (97.1) & $34 / 38(89.5)$ & 26/31 (83.9) & 93/103 (90.3) & 15 \\
\hline Gentamicin & 10/39 (25.6) & 17/44 (38.6) & 19/33 (57.6) & 46/116 (39.7) & 2 \\
\hline Imipenem & $34 / 34(100.0)$ & $39 / 43(90.7)$ & 28/33 (84.8) & 101/110 (91.8) & 8 \\
\hline Meropenem & 40/41 (97.6) & $39 / 43(90.7)$ & 28/33 (84.8) & 108/11792.3 & 1 \\
\hline Piperacillin-Tazobactam & 23/41 (56.1) & 21/39 (53.8) & $17 / 30(56.7)$ & $61 / 11055.5$ & 8 \\
\hline \multicolumn{6}{|c|}{ Gram-negative non-fermenters } \\
\hline Amikacin & $16 / 21(76.2)$ & 9/17 (52.9) & $1 / 15(6.7)$ & $26 / 53(49.1)$ & 11 \\
\hline Ceftazidime & $1 / 21(4.8)$ & $7 / 21(33.3)$ & $1 / 16(6.3)$ & 9/59 (15.3) & 6 \\
\hline Ciprofloxacin & $5 / 22(22.7)$ & $6 / 22(27.3)$ & $1 / 17(5.9)$ & 12/61 (19.7) & 3 \\
\hline Gentamicin & 2/22 (9.1) & 3/17 (17.6) & $1 / 13(7.7)$ & 6/52 (11.5) & 12 \\
\hline Imipenem & $2 / 16(12.5)$ & $5 / 20(25)$ & 0/16 (0.0) & $7 / 52(13.5)$ & 12 \\
\hline Meropenem & $5 / 22(27.2)$ & $5 / 20(25.0)$ & $0 / 16(0.0)$ & 10/58 (17.2) & 6 \\
\hline Piperacillin-Tazobactam & $4 / 22(18.2)$ & $4 / 20(20.0)$ & $1 / 16(6.3)$ & $6 / 58(15.5)$ & 6 \\
\hline \multicolumn{6}{|l|}{ Fungal isolates } \\
\hline Fluconazole & $2 / 9(22.5)$ & $7 / 10(70.0)$ & 18/12 (54.8) & 17/31 (54.8) & 0 \\
\hline
\end{tabular}

${ }^{\mathrm{a} O m i t t e d ~ d u e ~ t o ~ m i s s i n g ~ d a t a ~}$

ESBL organisms formed the majority of the MDROs in the unit $(60 / 681 ; 9 \%)$ and were comprised of K. pneumoniae $(52 / 681 ; 8 \%)$, with a minority of ESBL E. coli $(8 / 681$; $1 \%)$. Extensively-drug resistant (XDR) A. baumannii constituted $7.0 \%$ of the total resistance observed in the study. Overall, CREs accounted for 1.4\% (10/681) of MDROs.

Most S. aureus isolates were MRSA (19/24; 79.0\%) which comprised $3.0 \%(24 / 681)$ of the total study population.

When MDR organisms were analysed, a statistically significant decrease in all ESBL organisms was noted between 2014 (70.0\%) and 2018 (36.4\%) (OR 0.24; 95\% CI $[0.09,0.65] ; p=0.005)$.
An increase in XDR isolates between 2014 (75.0\%) and 2018 (88.9\%) was detected, however, this was not of statistical significance (OR 2.67; 95\% CI $[0.47,15.14] ; p=$ $0.3)$.

Comparisons of CRE samples from 2014 (2.5\%) to 2018 (15.2\%) (OR 6.96; 95\% CI [0.77, 62.93]; $p=0.08$ ) demonstrated a non-significant increase in the number of cases.

\section{Discussion}

This study represents the first published microbial profile of neonatal sepsis at a tertiary/quaternary unit in KwaZulu-Natal, South Africa. A preponderance of LOS 


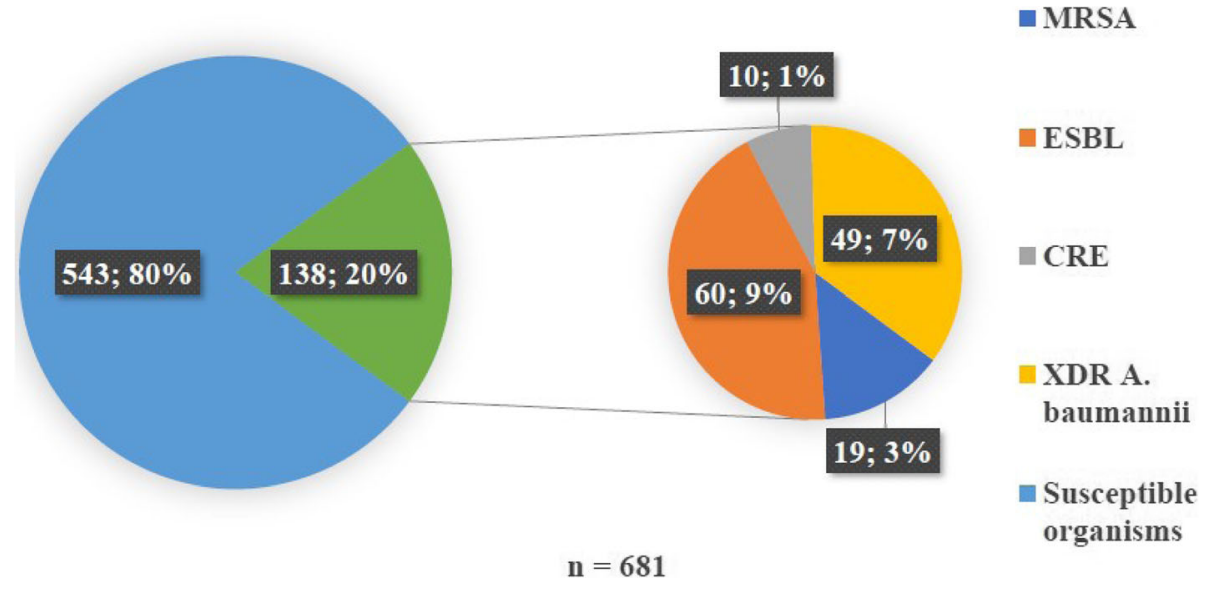

Fig. 2 Multidrug resistant organisms during the study period

versus EOS was observed. The leading organisms were CoNS, followed by $K$. pneumoniae, A. baumannii and $E$. faecium. A decrease in susceptibility to first- and second-line antibiotics, in gram-positives, gramnegatives and fungi was demonstrated. However, susceptibility to broad spectrum antibiotics such as vancomycin for gram positives and carbapenems for Enterobacterales was maintained. ESBL $K$. pneumoniae and XDR Acinetobacter baumannii represented the predominant MDR types.

Tertiary NICU settings, such as the NICU at IALCH, treat neonates that are mostly premature and of a low birth weight [22]. According to Giannoni et al. (2018), hospital-acquired LOS was higher in preterm infants when compared to EOS [23]. A significant majority of late-onset neonatal sepsis (86.8\%) was observed in our study which corroborated with other studies from tertiary level neonatal units in South African where LOS accounted for 83.0 to $93.0 \%$ of cases [10-12].

Studies concluded that almost $70.0 \%$ of first-onset infections in LOS were caused by gram-positive organisms, followed by gram-negatives (18.0\%) and fungi (12.0\%) which is supported by the findings of this study [24]. Apart from CoNS, the leading organisms were enterococci, K. pneumoniae and A. baumannii. These findings are consistent with reports from other African countries and India $[13,14,25]$. Despite the evidence surrounding CoNS as pathogens of neonatal sepsis, isolates may still represent blood culture contamination as skin colonisers [26]. The absence of clinical data in this study rendered determining clinical significance challenging. Of note, studies from other low-and-middle income countries have also demonstrated that CoNS were leading pathogens of neonatal sepsis after adjusting for contamination $[8,10,11,13,15,25]$.

Studies from Botswana and South Africa have documented enterococci as a leading cause of gram-positive sepsis $(12.2$ to $18.0 \%)[15,16]$. In the current study, enterococci emerged as significant pathogens of grampositive sepsis with a predominance of E. faecium in LOS and E. faecalis in EOS. The possibility remains that antibiotic selective pressure may drive the shift from ampicillin-susceptible E. faecalis in EOS to ampicillinresistant $E$. faecium in LOS. This species-specific differentiation requires confirmation with larger studies.

South African studies determined K. pneumoniae and $S$. aureus are leading pathogens of neonatal sepsis [11, 12]. However, in this study, S. aureus was not a common cause of sepsis. Among Enterobacterales, K. pneumoniae was isolated most frequently, alongside other organisms such as E. coli. Studies confirm that E. coli and K. pneumoniae are well-recognized pathogens of neonatal sepsis [8, 9, 27]. Additionally, our study found that nonfermenters such as A. baumannii, S. maltophilia and $P$. aeruginosa were also causes of sepsis, which was supported by other reports [28, 29].

Though overall rates of candidaemia remained low when compared to bacteraemia, C. parapsilosis was a notable cause of neonatal candidaemia, an observation corroborated by findings from other South African studies $[16,30]$.

Currently, ampicillin, cloxacillin and vancomycin are advocated in the treatment regimen of gram-positive organisms. Staphylococcus aureus and CoNS showed high resistance to cloxacillin, which has been demonstrated both globally and in South Africa $[10,25,31]$. In addition, due to the prevalence of E. faecium within the NICU, ampicillin susceptibility was low, which was also described in another South African setting [10].

It has been observed that resistance amongst the gram-negative population is on the rise within NICUs [32]. This study confirms these findings, as gramnegative organisms in this unit demonstrated high levels of resistance to first-line antibiotics (i.e., cefotaxime and 
gentamicin). However, susceptibility to broaderspectrum agents, which included carbapenems and amikacin, was evident. Other research has reported high levels of resistance to antimicrobials used to treat gramnegatives, including ampicillin, ceftazidime, cefotaxime, imipenem, gentamicin, ciprofloxacin while maintaining susceptibility to meropenem, which was confirmed by our analysis [33]..

Neonatal units have demonstrated variable fluconazole susceptibility patterns that are dependent upon the species of their predominating fungal pathogens [34, 35]. The administration of fluconazole prophylaxis to highrisk patients, which is the practice within this NICU, may lead to selection of fluconazole resistant species [32]. Approximately half of all candida isolates, especially C. parapsilosis, were resistant to fluconazole and this finding is supported by another South African study that reported fluconazole resistance amongst $C$. parapsilosis of $54.0 \%$ [7].

When susceptibility trends over the three study periods were compared for all antibiotics, susceptibility of cefotaxime amongst Enterobacterales increased significantly during the study period, although it remained below $50.0 \%$. This observation may be attributed to the decreasing levels of ESBL organisms. There were no statistically significant downward trends in susceptibility apart from amikacin. Literature includes mixed reports regarding the susceptibility of aminoglycosides, such as amikacin and gentamicin. Roy et al. (2017), reported increased amikacin resistance in an Indian NICU over a 15-year period [36]. In contrast, low levels of amikacin resistance have been documented in other studies; therefore, amikacin is frequently included in empiric regimens [37]. The temporal increase in susceptibility of gentamicin found in this study, may be attributed to the preferential use of amikacin, instead of gentamicin, in the unit. This occurrence requires further observation over time.

Despite the global trend towards increasing MDRO, the rates of ESBLs in our study have undergone a statistically significant decrease from 2014 to 2018, which is in keeping with the increased cefotaxime susceptibilities observed. Changes in ESBL rates may also be attributed to the increase of other MDROs within the unit. XDR $A$. baumannii were important isolates during the study period that showed an increased occurrence, albeit not statistically significant. This study also demonstrated the emergence of CRE, which has been implicated in neonatal sepsis in a recent South African study [33]. Although MRSA has been found to cause neonatal sepsis in other South African studies, the overall contribution of MRSA to the study population was lower than described in other South Africa neonatal settings $[10,12,16]$.

According to the World Health Organization (WHO), first line antibiotic therapy for neonatal sepsis consists of benzylpenicillin/ampicillin and gentamicin [5]. Choosing an appropriate empiric antimicrobial regimen in the IALCH NICU remains a challenge. Amoxicillinclavulanate and piperacillin-tazobactam as first-line agents for EOS and LOS, respectively, currently used in this NICU, are inappropriate. In view of the high levels of antimicrobial resistance observed, meropenem with or without vancomycin provides optimal empiric cover for both EOS and LOS. The addition of vancomycin would depend on the presence of risk factors for staphylococcal or E. faecium infection such as the presence of intraabdominal sepsis. This regimen would be effective due to the high prevalence of ESBL organisms and resistant gram-positive organisms in the neonatal unit. However, it would not be active against XDR A. baumannii. Therefore, in clinically unstable cases with suspected preliminary Acinetobacter species, as suggested by the gram stain and MDR appearance on direct Kirby-Bauer disk diffusion, appropriate combination therapy with collaboration between clinician and clinical microbiologists is suggested. This approach would be on an individual basis and would require careful clinically correlation due to the inherent limitations of direct disk diffusion testing. Newer antimicrobials are available for the management of sepsis. However, further investigation into use in the neonatal population is required. For empiric treatment for candidiasis, amphotericin B is recommended, which correlates with recommendations issued by the Infectious Diseases Society of America [38].

Apart from using empiric broad-spectrum antimicrobials in the unit, effective infection control practices need to be enforced in curb the spread of MDROs. Consequently, several infection control measures are in effect within the unit such strict hand hygiene practices, isolation and cohorting of patients, intensive environmental cleaning, individual-use equipment, and the placement of a sentry at the entrance of the unit to monitor handwashing and wearing of personal protective equipment of all those who enter the unit. These measures have been in effect during the study period. In addition, antimicrobial stewardship practices are utilised, which include the de-escalation of therapy in conjunction with microbiological confirmation and the discontinuation of antimicrobials based on clinical, haematological, and microbiological grounds. Decisions regarding antimicrobials are made jointly with the microbiologist and the attending clinician.

When assessing the appropriateness of the above recommendations, the study's limitations need to be considered. This study is based on laboratory surveillance and clinical data was not collected. However, clinical correlation is required to assist decisions regarding clinical relevance of potential pathogens in blood cultures, especially for CoNS. Results cannot be generalised to 
other settings, hospitals, or patient profiles as a single centre was utilised for the analysis. Premature neonates were not stratified within the study population and may present a different microbial profile than other groups of neonates. This study may be underpowered to determine temporal fluctuations amongst less frequently occurring organisms. Lastly, some antimicrobial data was unavailable.

\section{Conclusion}

First-line antimicrobials, advocated by the WHO for treatment of neonatal sepsis, have proven ineffective in this unit due to high levels of AMR. Gram-positive sepsis, caused by CoNS and enterococci, was the leading cause of sepsis in this study. Gram-negative sepsis occurs to a lesser extent and is mainly comprised of XDR A. baumannii and ESBL $K$. pneumoniae. These MDROs create a therapeutic challenge and require broadspectrum agents or combination therapy in consultation with paediatric infectious disease specialists and clinical microbiologists. The resistance noted in fungal isolates also necessitates broad-spectrum antifungals. Therefore, surveillance of the microbial profile of neonatal sepsis provides data to develop an appropriate empiric regimen and antimicrobial stewardship activities, which would ultimately improve sepsis outcomes.

\section{Abbreviations \\ AMR: Antimicrobial resistance; CoNS: Coagulase-negative staphylococci; CRE: Carbapenem-resistant Enterobacterales; EOS: Early-onset sepsis; ESBL: Extended-spectrum beta-lactamase; GBS: Group B streptococcus; HIC: High-income country; IALCH: Inkosi Albert Luthuli Central Hospital; LOS: Late onset sepsis; MDR: Multi-drug resistant; MDRO: Multidrug resistant organism; MRSA: Methicillin-resistant Staphylococcus aureus; NICU: Neonatal intensive care unit; OR: Odds ratio; VRE: Vancomycin resistant enterococci; WHO: World Health Organization; XDR: Extensively-drug resistant}

\section{Acknowledgements}

To Ms. Cathy Connolly, for her invaluable experience in biostatistics. To the National Health Laboratory Service, for allowing access to their database.

\section{Authors' contributions}

DP's contribution included conceptualisation of the project, development of the methodology and protocol and liaison/applications for ethical clearance and relevant consents, data curation, formal data analysis and writing of the manuscript. YM performed the role of project supervisor, including synthesis of the protocol, overseeing editing and review of the manuscript. LN and $\mathrm{KSH}$ contributed to editing and reviewing the manuscript. All authors have reviewed and approved the manuscript.

\section{Funding}

This research did not receive any specific grant from funding agencies in the public, commercial, or not-for-profit sectors.

\section{Availability of data and materials}

The data that support the findings of this study are available from the National Health Laboratory Service (Sandringham, South Africa) but restrictions apply to the availability of these data, which were used under license for the current study, and so are not publicly available. Data are however available from the authors upon reasonable request and with permission of the National Health Laboratory Service (Sandringham, South Africa).

\section{Ethics approval and consent to participate}

This study has received approval from the University of KwaZulu-Natal Biomedical Research Ethics Committee (BE019/19), National Health Laboratory Service, Inkosi Albert Luthuli Central Hospital, and the KwaZulu-Natal Department of Health (South Africa). The National Health Laboratory Service granted permission and provided access to the raw data utilised in this study. The data was anonymised prior to use.

\section{Consent for publication}

Not applicable.

\section{Competing interests}

The authors declare that they have no competing interests.

\section{Author details}

'Department of Medical Microbiology, National Health Laboratory Service, Inkosi Albert Luthuli Central Hospital, 800 Vusi Mzimela Road, Durban, KwaZulu-Natal 4091, South Africa. ${ }^{2}$ School of Laboratory Medicine and Medical Sciences, Nelson R. Mandela School of Medicine, University of KwaZulu Natal, 716 Umbilo Road, Berea, KwaZulu-Natal 4001, South Africa. ${ }^{3}$ Neonatal Intensive Care Unit, Inkosi Albert Luthuli Central Hospital, 800 Vusi Mzimela Road, Durban, KwaZulu-Natal 4091, South Africa. ${ }^{4}$ Department of Paediatrics and Child Health, Nelson R Mandela School of Medicine, University of KwaZulu Natal, 716 Umbilo Road, Berea, KwaZulu-Natal 4001, South Africa.

Received: 15 August 2020 Accepted: 3 February 2021

Published online: 27 February 2021

\section{References}

1. World Health Organization. Antimicrobial resistance: global report on surveillance: World Health Organization; 2014.

2. Li G, Bielicki JA, Ahmed ANU, Islam MS, Berezin EN, Gallacci CB, et al. Towards understanding global patterns of antimicrobial use and resistance in neonatal sepsis: insights from the NeoAMR network. Arch Dis Child. 2020; 105(1):26-31.

3. Liu L, Oza S, Hogan D, Chu Y, Perin J, Zhu J, et al. Global, regional, and national causes of under-5 mortality in 2000-15: an updated systematic analysis with implications for the Sustainable Development Goals. Lancet. 2016;388(10063):3027-35.

4. Ranjeva SL, Warf BC, Schiff SJ. Economic burden of neonatal sepsis in subSaharan Africa. BMJ Glob Health. 2018;3(1):e000347. https://doi.org/10.1136/ bmjgh-2017-000347 Accessed on 19 Sept 2018.

5. Fuchs A, Bielicki J, Mathur S, Sharland M, Van Den Anker J. Antibiotic use for sepsis in neonates and children: 2016 evidence update. WHO Reviews 2016.

6. Coetzee M, Mbowane N, de Witt T. Neonatal sepsis: Highlighting the principles of diagnosis and management. S Afr J Child Health. 2017;11(2): 99-103.

7. Shane AL, Stoll B. Neonatal sepsis: progress towards improved outcomes. Journal of Infection. 2014;68:24-32.

8. Pokhrel B, Koirala T, Shah G, Joshi S, Baral P. Bacteriological profile and antibiotic susceptibility of neonatal sepsis in neonatal intensive care unit of a tertiary hospital in Nepal. BMC Pediatr. 2018;18(1):208.

9. Muley VA, Ghadage DP, Bhore AV. Bacteriological profile of neonatal septicemia in a tertiary care hospital from Western India. J Global Infect Dis. 2015;7(2):75.

10. Ballot DE, Nana T, Sriruttan C, Cooper PA. Bacterial bloodstream infections in neonates in a developing country. ISRN Pediatr. 2012:508512. https://doi. org/10.5402/2012/5085122012.

11. Motara F, Ballot D, Perovic O. Epidemiology of neonatal sepsis at Johannesburg Hospital. South Afr J Epidemiol Infect. 2005;20(3):90-3.

12. Lebea MM, Davies V. Evaluation of culture-proven neonatal sepsis at a tertiary care hospital in Johannesburg, South Africa. S Afr J Child Health. 2017;11(4):170-3.

13. Aku FY, Akweongo P, Nyarko K, Sackey S, Wurapa F, Afari EA, et al. Bacteriological profile and antibiotic susceptibility pattern of common isolates of neonatal sepsis, Ho Municipality, Ghana-2016. Matern Health Neonatol Perinatol. 2018;4(1):2.

14. El-Din S, Rabie EM, El-Sokkary MMA, Bassiouny MR, Hassan R. Epidemiology of neonatal sepsis and implicated pathogens: a study from Egypt. Biomed Res Int. 2015;2015:1-11. https://doi.org/10.1155/2015/509484. 
15. Mpinda-Joseph P, Anand Paramadhas BD, Reyes G, Maruatona MB, Chise M, Monokwane-Thupiso BB, et al. Healthcare-associated infections including neonatal bloodstreamFshe infections in a leading tertiary hospital in Botswana. Hosp Pract. 2019;47(4):203-10.

16. Dramowski A, Madide A, Bekker A. Neonatal nosocomial bloodstream infections at a referral hospital in a middle-income country: burden, pathogens, antimicrobial resistance and mortality. Paediatr Int Child Health. 2015:35(3):265-72.

17. University of California San Francisco (UCSF) Children's Hospital. Candidiasis in the newborn. In: Intensive Care Nursery House Staff Manual; 2004. p. 128-9. Accessed on 25 Apr 2020 from https://www.ucsfbenioffchildrens.org.

18. Ballot DE, Bosman N, Nana T, Ramdin T, Cooper PA. Background changing patterns of neonatal fungal sepsis in a developing country. J Trop Pediatr. 2013;59(6):460-4.

19. Cole BK, Ilikj M, McCloskey CB, Chavez-Bueno S. Antibiotic resistance and molecular characterization of bacteremia Escherichia coli isolates from newborns in the United States. PLoS One. 2019;14(7):e0219352. https://doi. org/10.1371/journal.pone.0219352 Accessed on 16 Apr 2020.

20. Crichton $\mathrm{H}, \mathrm{O}^{\prime}$ Connell N, Rabie H, Whitelaw A, Dramowski A. Neonatal and paediatric bloodstream infections: Pathogens, antimicrobial resistance patterns and prescribing practice at Khayelitsha District Hospital, Cape Town, South Africa. S Afr Med J. 2018;108(2):99-104.

21. Magiorakos AP, Srinivasen A, Carey RB, et al. Multidrug-resistant, extensively drug-resistant and pandrug-resistant bacteria: an international expert proposal for interim standard definitions for acquired resistance. Clin Microbiol Infect. 2012;18(3):268-81.

22. Vergnano S, Sharland M, Kazembe P, Mwansambo C, Heath P. Neonatal sepsis: an international perspective. Arch Dis Child Fetal Neonatal Ed. 2005; 90(3):220-4.

23. Giannoni E, Agyeman PKA, Stocker M, Posfay-Barbe KM, Heininger U, Spycher BD, et al. Neonatal Sepsis of Early Onset, and Hospital-Acquired and Community-Acquired Late Onset: A Prospective Population-Based Cohort Study. J Pediatr. 2018;201:106-14.

24. Cortese F, Scicchitano P, Gesualdo M, Filaninno A, De Giorgi E, Schettini F, et al. Early and Late Infections in Newborns: Where Do We Stand? A Review. Pediatr Neonatol. 2016;57(4):265-73.

25. Labi AK, Obeng-Nkrumah N, Bjerrum S, Enweronu-Laryea C, Newman MJ. Neonatal bloodstream infections in a Ghanaian Tertiary Hospital: Are the current antibiotic recommendations adequate? BMC Infect Dis. 2016;16(1): 598.

26. Souvenir D, Anderson DE, Palpant S, Mroch H, Askin S, Anderson J, et al. Blood Cultures Positive for Coagulase-Negative Staphylococci: Antisepsis, Pseudobacteremia, and Therapy of Patients. J Clin Microbiol. 1998;36(7): 1923.

27. Cutland CL. Epidemiology and prevention of sepsis in young infants and the potential impact of maternal HIV infection on neonatal sepsis. 2016. Accessed from: http://hdl.handle.net/10539/22516 on 12 January 2020

28. Thomas R, Wadula J, Seetharam S, Velaphi S. Prevalence, antimicrobial susceptibility profiles and case fatality rates of Acinetobacter Baumannii sepsis in a neonatal unit. J Infect Dev Ctries. 2018;12(4):211-9.

29. Viswanathan $R$, Singh AK, Mukherjee S, Mukherjee R, Das P, Basu S. Aetiology and antimicrobial resistance of neonatal sepsis at a tertiary care centre in eastern India: a 3 year study. Indian J Pediatr. 2011;78(4):409-12.

30. Govender NP, Patel J, Magobo RE, Naicker S, Wadula J, Whitelaw A, et al. Emergence of azole-resistant Candida parapsilosis causing bloodstream infection: results from laboratory-based sentinel surveillance in South Africa. J Antimicrob Chemother. 2016;71(7):1994-2004.

31. Morkel G, Bekker A, Marais B, Kirsten G, Van Wyk J, Dramowski A. Bloodstream infections and antimicrobial resistance patterns in a South African neonatal intensive care unit. Paediatr Int Child Health. 2014;34(2): 108-14.

32. Patel SJ, Saiman L. Antibiotic resistance in neonatal intensive care unit pathogens: mechanisms, clinical impact, and prevention including antibiotic stewardship. Clin Perinatol. 2010;37(3):547-63.

33. Ballot DE, Bandini R, Nana T, Bosman N, Thomas T, Davies VA, et al. A review of -multidrug-resistant Enterobacteriaceae in a neonatal unit in Johannesburg, South Africa. BMC Pediatr. 2019;19(1):320.

34. Blyth CC, Chen SCA, Slavin MA, Serena C, Nguyen Q, Marriott D, et al. Not Just Little Adults: Candidemia Epidemiology, Molecular Characterization, and Antifungal Susceptibility in Neonatal and Pediatric Patients. Pediatrics. 2009; 123(5):1360.
35. Goel N, Ranjan PK, Aggarwal R, Chaudhary U, Sanjeev N. Emergence of nonalbicans Candida in neonatal septicemia and antifungal susceptibility: experience from a tertiary care center. J Lab Physicians. 2009;1(2):53-5.

36. Roy MP, Bhatt M, Maurya V, Arya S, Gaind R, Chellani HK. Changing trend in bacterial etiology and antibiotic resistance in sepsis of intramural neonates at a tertiary care hospital. J Postgrad Med. 2017;63(3):162-8.

37. Sharma CM, Agrawal RP, Sharan H, Kumar B, Sharma D, Bhatia SS. "Neonatal Sepsis": Bacteria and their Susceptibility Pattern towards Antibiotics in Neonatal Intensive Care Unit. J Clin Diagn Res. 2013;7(11):2511-3.

38. Pappas PG, Kauffman CA, Andes DR, Clancy CJ, Marr KA, Ostrosky-Zeichner L, et al. Clinical practice guideline for the management of candidiasis: 2016 update by the Infectious Diseases Society of America. Clin Infect Dis. 2016; 62(4):e1-e50. https://doi.org/10.1093/cid/civ933 Accessed 02 Jan 2020.

\section{Publisher's Note}

Springer Nature remains neutral with regard to jurisdictional claims in published maps and institutional affiliations.
Ready to submit your research? Choose BMC and benefit from:

- fast, convenient online submission

- thorough peer review by experienced researchers in your field

- rapid publication on acceptance

- support for research data, including large and complex data types

- gold Open Access which fosters wider collaboration and increased citations

- maximum visibility for your research: over $100 \mathrm{M}$ website views per year

At BMC, research is always in progress.

Learn more biomedcentral.com/submissions 